\title{
El manejo del suelo salino usando arena afecta el crecimiento de raíces y la sobrevivencia de plántulas de Prosopis tamarugo Phil. (Fabaceae)
}

\section{Management saline soil using sand affects growth of roots and increases seedling survival Prosopis tamarugo Phil. (Fabaceae)}

\author{
Mario F. León ${ }^{1}$, Sergio I. Silva ${ }^{1}$, Ana Sandoval $^{1}$, Ismael Aracena ${ }^{2}$, Francisco Quiñones ${ }^{2}$ \& Pedro \\ LEÓN-LOBOS ${ }^{1^{*}}$
}

'Banco Base de Semillas, Instituto de Investigaciones Agropecuarias, INIA Intihuasi, Camino a Peralillo s/n, km 3 de Vicuña, Casilla 73, Vicuña, Chile.

${ }^{2}$ Sociedad Química y Minera de Chile S.A., Aníbal Pinto 3228, Antofagasta, Chile.

*pleon@inia.cl

\begin{abstract}
RESUMEN
La regeneración natural del árbol endémico Prosopis tamarugo es casi inexistente en la Pampa del Tamarugal, probablemente la salinidad del suelo es una barrera para el establecimiento de plántulas. Aunque existen numerosas técnicas para manejar la salinidad del suelo, la creación de un sustrato en la que suelo salino es mezclado con arena es escasamente conocido. En el presente estudio se profundizó en el efecto de tres sustratos experimentales (suelo salino natural, suelo 1:1 y suelo 1:2) en el crecimiento de raíces y supervivencia de plántulas de $P$. tamarugo durante un período de 50 días. El suelo 1:1 fue creado mezclando suelo natural y arena en igual proporción y el suelo 1:2, aumentado la proporción de arena al doble. Para los tres suelos se caracterizó la densidad aparente (D.A.), conductividad eléctrica (C.E.) y la concentración de sodio y cloruro. El crecimiento radicular de las plántulas fue caracterizado midiendo al final del estudio la longitud y biomasa de raíces, cociente raíz/tallo $(\mathrm{R} / \mathrm{T})$ y longitud radicular específica (LRE), además se monitoreó la biomasa radicular durante el estudio. El crecimiento aéreo se midió a través de la formación de hojas. Finalmente fue determinado el contenido de sales en los tejidos y la sobrevivencia de plántulas en cada suelo experimental. El suelo natural presentó una C.E. (176 $\mathrm{dS} / \mathrm{m})$ y D.A. (0,9 g/cc) más alta comparada con el suelo $1: 1(132 \mathrm{dS} / \mathrm{m}$ y $1,1 \mathrm{~g} / \mathrm{cc})$ y suelo $1: 2(140 \mathrm{dS} / \mathrm{m} \mathrm{y} 1,4 \mathrm{~g} / \mathrm{cc})$. La concentración de sodio y cloruro disminuyó en promedio un 37 y 41\% en los suelos 1:1 y 1:2, respectivamente comparado con el suelo salino natural. Las plántulas que crecieron en estos dos suelos presentaron un crecimiento significativo en longitud y biomasa radicular, R/T y LRE e incrementaron notablemente su biomasa radicular a partir del día 30. También exhibieron un menor contenido de sodio y cloruro en sus tejidos, mayor número de pares de hojas y una alta sobrevivencia final. Estos resultados apoyan la idea que incorporando arena en suelo salino como técnica de manejo de la salinidad del suelo favorece el crecimiento de las raíces y aumenta la supervivencia de plántulas de P. tamarugo.
\end{abstract}

Palabras Clave: Tamarugo, raíces, suelo, sodio y cloruro, conductividad eléctrica.

\begin{abstract}
Natural regeneration of endemic tree Prosopis tamarugo in the Pampa del Tamarugal is almost absent. Soil salinity is probably an important barrier for tamarugo seedling establishment. Despite there are numerous techniques to manage soil salinity, substrates prepared using mix of saline soil and sand are poorly known. In the present study, the effect of different experimental substrates was evaluated on root growth and seedling survival of $P$. tamarugo. The experimental soils were prepared using natural soil and sand in equal proportion (1:1) and using a double proportion of sand (1:2). Saline soil used as control treatment. Bulk density (B.D.), electrical conductivity (E.C.) and sodium and chloride concentration was characterized for the three experimental soils. Seedling root growth was evaluated through length and root biomass, root/ shoot ratio $(\mathrm{R} / \mathrm{S})$ and specific root length (SRL). Root biomass was also monitored during the study. Aerial growth was determined using leaf formation. Additionally, salt content in tissues and survival of seedlings were evaluated at the day 50 of initiated of experiment. The natural soil presented a E.C. $(176 \mathrm{dS} / \mathrm{m})$ and B.D. $(0.9 \mathrm{~g} / \mathrm{cc})$ higher tan experimental soils $1: 1(132 \mathrm{dS} / \mathrm{m}$ and $1.1 \mathrm{~g} / \mathrm{cc})$ and $1: 2(140 \mathrm{dS} / \mathrm{m}$ and $1.4 \mathrm{~g} / \mathrm{cc})$. Sodium and chloride concentration decreased on average by 37 and $41 \%$ in $1: 1$ and 1:2 soils with sand amendment respectively in comparison with the natural saline soil. Seedlings grown in the two amendment soils showed significant growth in root length, root biomass, R/S and SRL and significantly increased their root biomass by day 30 . They also exhibited a lower sodium and chloride concentration in their tissues, more
\end{abstract}


number of pairs of leaves and a higher final survival compared with seedling growing on experimental natural saline soil. These results support the idea that amendment of saline soil with sand as a management technique promotes root growth and increases seedling survival of P. tamarugo.

KEYwORDs: Tamarugo, roots, soil, sodium and chloride, electrical conductivity.

\section{INTRODUCCIÓN}

Prosopis es un género con especies arbóreas y arbustivas que habitan las zonas áridas y semiáridas de América (Burkart 1976). De las especies en Chile, el árbol endémico En Peligro Prosopis tamarugo Phil. (Fabaceae) presenta una distribución restringida, en latitudes de 19 a $21^{\circ} \mathrm{S}$ y 750 a $1.100 \mathrm{~m}$ s.n.m. de las regiones del extremo norte (Ministerio Medio Ambiente 2013). En la Región de Iquique crecen pequeñas poblaciones naturales (Carevic et al. 2012, Ministerio Medio Ambiente 2013) que viven con casi nulos registros de precipitaciones, elevadas temperaturas y una intensa evapotranspiración en la gran llanura del Desierto de Atacama conocida como Pampa del Tamarugal (Houston 2006, Carevic 2014). El suelo es extremadamente salino y es usual la formación de una costra pétrea en cuyas oquedades hay acumulación de frutos y semillas y donde plántulas de $P$. tamarugo deben abrirse camino en su crecimiento. Éstas suelen tolerar una alta presencia de iones salinos en condiciones experimentales (Felker et al. 1981, Arce et al. 1990), pero en estado natural resulta difícil detectar su presencia. Se ha descrito que a partir de la ocurrencia de aluviones cordilleranos por intensas lluvias de verano, el acarreo de materiales (piedras, bolones y restos vegetales) rompe la superficie del suelo generando "micrositios de regeneración" y provocando arrastres de las sales de estos micrositios favoreciendo la germinación de semillas y el desarrollo de plántulas de P. tamarugo (Altamirano 2006, Ministerio Medio Ambiente 2013). En ausencia de estos eventos no resulta frecuente encontrar estados tempranos de P. tamarugo en la Pampa del Tamarugal, la alta salinidad del suelo es un factor que contribuiría a esta situación.

Rehabilitar un suelo salino es una tarea compleja y difícil de abordar. Ésta implica desafectar la influencia negativa de iones salinos que interactúan en la zona de influencia de las raíces de las plantas (Marschner 1995). Las raíces constituyen un indicador efectivo del efecto de la salinidad del suelo en las plantas debido a su extrema sensibilidad a la presencia de iones salinos, por ejemplo, las sales inhiben el crecimiento de raíces finas y producción de biomasa (Sinha \& Singh 1974, Mer et al. 2000, Imada et al. 2015) y afectan la adquisición de nutrientes minerales (Grattan \& Grieve 1992, Huy \& Schmidhalter 2005, Yang et al. 2015). Su influencia en los estados iniciales de $P$. tamarugo podría tener serias consecuencias para su regeneración natural, pero la información al respecto es escasa debido a la ausencia de plántulas in situ. En condiciones experimentales controladas se ha mostrado un efecto nocivo de las sales en la morfología y anatomía de las raíces de plántulas en otras especies de Prosopis (e.g., Serrato et al. 1992, Catalán et al. 1994, Reinoso et al. 2004, Hosseini Nasr et al. 2012). En general para las especies freatófitas, su arquitectura radicular implica el desarrollo profuso de sus raíces para explorar y obtener los recursos del suelo en desiertos, aspecto importante en las plántulas de $P$. tamarugo que asignan recursos a la formación de raíces que profundizan en el suelo. En su supervivencia en la Pampa del Tamarugal, P. tamarugo posee raíces pivotantes y superficiales que optimizan el uso de agua de los estratos profundos y nutrientes de los estratos superficiales del suelo, respectivamente (Mooney et al. 1980).

El manejo de la salinidad del suelo implica usar enmiendas (e.g., Gurbachan et al. 1989, Bradbury \& Ahmad 1990), incorporar en las raíces inóculos tolerantes a las sales (Dixon et al. 1993) o afectar las relaciones de intercambio de sales usando fertilizantes específicos (Hussain et al. 2015). En una vía menos común también es posible manejar el volumen de espacios porosos para facilitar el lavado progresivo de sales. En rigor, manejarlo para crear un suelo que posea alta densidad aparente para aumentar el drenaje del agua (Kramer \& Boyer 1995). Esto facilitaría el transporte de iones salinos hacia puntos alejados de la rizósfera de las plantas, análogo a la dilución de sales causada por los aluviones cordilleranos en la Pampa del Tamarugal. Rehabilitar el suelo salino hollado de los sectores de Zapiga, Salares Pintados-Bellavista y Salar de Llamara, que en algunos puntos alcanza una conductividad eléctrica de $182 \mathrm{dS} / \mathrm{m}$ (datos INIA, no publicados) y que conforman la Reserva Nacional Pampa del Tamarugal, es un paso necesario para una iniciativa real para la conservación de $P$. tamarugo en su hábitat. En el presente estudio profundizamos en el manejo del suelo salino en la supervivencia de plántulas de $P$. tamarugo analizando parámetros relacionados con las raíces. Predecimos que las plántulas exhiben una alta supervivencia cuando el crecimiento y desarrollo de sus raíces incrementa en un suelo que experimenta menor carga salina.

\section{MATERIALES Y MÉTODOS}

\section{Suelos eXPerimentales}

El suelo para el estudio provino de un sector del Salar de Bellavista, Reserva Nacional Pampa del Tamarugal y fue recolectado en 2014 a partir de tres calicatas de $1 \mathrm{~m}^{2}$ de diámetro y $0,8 \mathrm{~m}$ de profundidad. En cada calicata hubo que 
eliminar de su superficie una costra salina pétrea que osciló entre $15-20 \mathrm{~cm}$. Enseguida se recolectó el suelo que fue sometido a un análisis de salinidad en el extracto saturado (1:5) en el Laboratorio de Suelos, INIA Quilamapu. El suelo salino natural presentó una elevada conductividad eléctrica y una variedad de iones donde destacaron las concentraciones de sodio y cloruro tal como fue detectado en un análisis de suelo realizado en 2013 en diferentes sectores de la reserva (Tabla 1). En el presente estudio las concentraciones de sodio y cloruro estuvieron por sobre los $2000 \mathrm{mmol} / \mathrm{L}$ y se usaron como indicadores de la salinidad del suelo (Tabla 2). El suelo salino natural (control) fue secado en estufa (Heraeus Instruments D-63450) a $110^{\circ} \mathrm{C}$ durante $48 \mathrm{~h}$ (ASTM D221610). Una fracción de este suelo natural fue combinado homogéneamente con igual volumen de suelo arenoso seco comercial de $1 \mathrm{~mm}$ para producir el suelo experimental 1:1 y otra fue combinada con un volumen duplicado de arena para conformar el suelo experimental 1:2. Estos tres suelos experimentales fueron sometidos a un análisis de salinidad en el extracto saturado (1:5) y de densidad aparente y porosidad con el método del cilindro en el Laboratorio de Suelos, INIA Quilamapu (Tabla 2).

DISEÑO EXPERIMENTAL

Se usó un diseño experimental completamente al azar para establecer el efecto del suelo experimental en el crecimiento y sobrevivencia de plántulas de Prosopis tamarugo bajo condiciones de vivero. Cada suelo fue replicado en 88 unidades experimentales que consistió en un contenedor de $100 \mathrm{~cm}^{3}$ relleno con suelo experimental y distribuido en interspersión dentro de tres mesas de plantación $(1,5 * 3$ m) para cumplir el supuesto de independencia de los contenedores (Hurlbert 1984). En cada contenedor se sembró una semilla germinada de $P$. tamarugo y cada mesa de plantación fue regada con agua destilada con un nivel de $5 \mathrm{~mm}$ (1,2 litros) aplicado diariamente en la primera semana y posteriormente cada tres días hasta el final de la evaluación cuando se cumplieron 50 días. La germinación de las semillas fue realizada sometiendo las semillas a una escarificación de 15 min en ácido sulfúrico y luego manteniéndolas en cápsulas de Petri con agar-agar (1\%) dentro de una cámara de crecimiento (Binder KBWF-240) a $20{ }^{\circ} \mathrm{C}, 75 \%$ de humedad relativa y $12 / 12 \mathrm{~h}$ de fotoperiodo. Las semillas de Prosopis tamarugo usadas en el estudio pertenecen a las accesiones de tamarugo SQM-6 y SQM-7 que son preservadas en el Banco Base de Semillas (Centro Experimental Vicuña), INIA Intihuasi. Fueron recolectadas en abril 2011 de árboles que crecen en el Salar de Bellavista, Reserva Nacional Pampa del Tamarugal (Fig. 1). El estudio fue realizado en las dependencias del Banco de Semillas.

TABla 1. Caracterización de la salinidad del suelo de Reserva Nacional Pampa del Tamarugal realizada en 2013. Las letras en negritas son usadas para destacar los parámetros de conductividad eléctrica (C.E.) y los iones sodio y cloruro. Los valores son promedios $(1 \pm \mathrm{EE}, \mathrm{n}=$ 10 réplicas). / Characterization of soil salinity from National Reserve Pampa del Tamarugal conducted in 2013. Letters in bold for highlight the values of electrical conductivity and sodium and chloride ions. Values are mean ( $1 \pm \mathrm{SE}, \mathrm{n}=10$ replicates).

\begin{tabular}{ccccccccc}
\hline C.E. $(\mathrm{dS} / \mathrm{m})$ & Saturación extracto (\%) & \multicolumn{3}{c}{ Cationes $(\mathrm{mmol}(+) / \mathrm{L})$} & \multicolumn{3}{c}{ Aniones (mmol (-) / L) } \\
\hline & & $\mathrm{Ca}$ & $\mathrm{Mg}$ & $\mathrm{Na}$ & $\mathrm{K}$ & $\mathrm{Cl}$ & $\mathrm{SO}_{4}$ \\
\cline { 2 - 8 } 145,4 & 33,5 & 25,3 & 281,9 & 3728,8 & 103,4 & 2932,7 & 945.6 \\
\hline
\end{tabular}

TABLA 2. Caracterización físico-química de suelos experimentales previo a la siembra de semillas de Prosopis tamarugo. Los valores son promedios ( $1 \pm \mathrm{EE}, \mathrm{n}=10$ réplicas). Letras diferentes corresponden a diferencias significativas (Comparaciones múltiples t-test, $\mathrm{P}<0,001)$. Densidad aparente (DA), conductividad eléctrica (CE). Los valores en la tabla para la arena son presentados como referencia. / Physicalchemical characterization of experimental soils prior to sowing seed Prosopis tamarugo. Values are mean $(1 \pm \mathrm{SE}, \mathrm{n}=10 \mathrm{replicates})$. Different letters correspond to significant differences ( $\mathrm{t}$-test multiple comparisons, $\mathrm{P}<0.001$ ). Bulk density (DA), electrical conductivity (CE). The values in the table for sand soil are presented for reference.

\begin{tabular}{|c|c|c|c|c|c|}
\hline SUELOS EXPERIMENTALES & $\mathrm{DA}(\mathrm{g} / \mathrm{cc})$ & Porosidad $(\%)$ & $\mathrm{CE}(\mathrm{dS} / \mathrm{m})$ & \multicolumn{2}{|c|}{ IONES $(\mathrm{mmol} / \mathrm{L})$} \\
\hline & & & & $\mathrm{Na}$ & $\mathrm{Cl}$ \\
\hline Suelo natural & $0,9^{\mathrm{a}} \pm 0,02$ & $67,4^{\mathrm{a}} \pm 0,7$ & $176,3^{\mathrm{a}} \pm 2,3$ & $2333^{\mathrm{a}} \pm 119,7$ & $4143^{a} \pm 179,9$ \\
\hline Suelo 1:1 & $1,1^{\mathrm{b}} \pm 0,01$ & $58,8^{\mathrm{b}} \pm 0,4$ & $132,2^{b} \pm 1,2$ & $1482^{\mathrm{b}} \pm 64,4$ & $2327^{\mathrm{b}} \pm 38,1$ \\
\hline Suelo 1:2 & $1,4^{\mathrm{c}} \pm 0,03$ & $46,0^{\mathrm{c}} \pm 1,0$ & $140.0^{\mathrm{b}} \pm 7,1$ & $1479^{b} \pm 114,8$ & $2531^{b} \pm 224,9$ \\
\hline Arena & $1,4 \pm 0,01$ & $47,1 \pm 2,3$ & $0,6 \pm 0,0$ & $1,1 \pm 0,0$ & $0,8 \pm 0,1$ \\
\hline
\end{tabular}


CReCimiento DE LAS RAíces

Se evaluó el crecimiento de las raíces de las plántulas a los 50 días midiendo la longitud radicular, biomasa seca de las raíces, relación raíz/tallo y longitud radicular específica. También se evaluó el crecimiento de las raíces determinando el cambio en el incremento de la biomasa radicular $(\Delta)$ a $\operatorname{los} 20,30$ y 40 días. En cada caso se seleccionaron aleatoriamente 10 contenedores cuyas plántulas fueron liberadas del sustrato y cuidadosamente lavadas con agua corriente dentro de un tamiz de $250 \mu \mathrm{m}$ para evitar pérdida de raíces finas. Se midió la longitud de raíz usando un pie de metro digital $( \pm 0,01 \mathrm{~mm})$. La biomasa seca se estimó separando las muestras de tallo y raíz y secando en una estufa a $75^{\circ}$ por $48 \mathrm{~h}$ (Sadzawka et al. 2007) antes de ser pesadas en una balanza analítica $( \pm 0,0001 \mathrm{~g})$. El cociente raíz / tallo fue obtenido dividiendo la masa seca de la raíz por la suma de la masa seca de tallo y hojas. La longitud radicular específica fue obtenida dividiendo la longitud por la masa seca de la raíz. Tanto el cociente raíz / tallo como la longitud radicular específica fueron usados como indicadores de calidad del suelo para el crecimiento de las plántulas.
Mortalidad DE PLÁNTULAS Y CRECIMIENTO AÉREO EN LOS SUELOS EXPERIMENTALES

La mortalidad de plántulas fue evaluada a los 50 días, contabilizando el número total de plántulas muertas en cada suelo experimental. El crecimiento aéreo de las plántulas se evaluó monitoreando la formación de hojas como indicador de este crecimiento. Para esto se seleccionaron al azar 30 plántulas por cada suelo experimental y se contabilizó el número de pares de hojas verdaderas formadas en el período de 50 días.

Salinidad En los tejidos de las Plántulas

Para establecer la efectividad de los suelos experimentales en el desempeño de las plántulas de $P$. tamarugo se evaluó el contenido de sales presentes en sus tejidos al final del estudio. Para esto se preparó una muestra de material vegetal usando diez plántulas sobrevivientes por cada suelo experimental. El pool de plántulas fue secado en una estufa a $75^{\circ}$ por $48 \mathrm{~h}$ (Sadzawka et al. 2007) y molido a polvo fino. El material resultante fue dividido en tres submuestras que fueron usadas para determinar la concentración de iones sodio y cloruro en el Laboratorio de Análisis Foliar, Centro Experimental Vicuña, INIA Intihuasi.
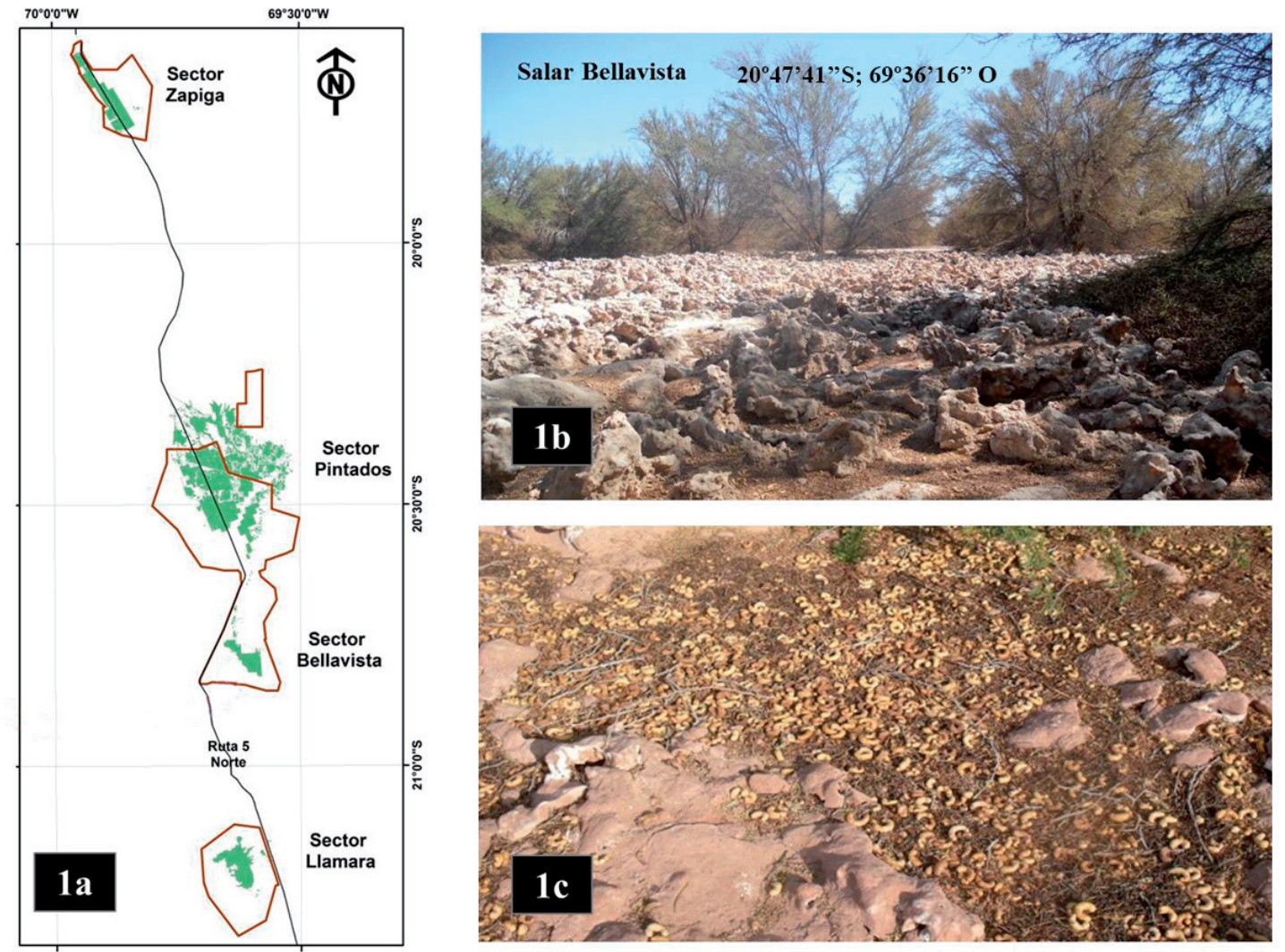

Figura 1a. Ubicación geográfica de Reserva Nacional Pampa del Tamarugal (polígonos rojos) y sus cuatro sectores. 1b, vista parcial de costra salina y 1c, frutos de tamarugo dispersos sobre la costra salina. / Geographical location of Reserva Nacional Pampa del Tamarugal (red polygons) and its four sectors. 1b) partial view of salt crust and 1c) tamarugo fruits scattered on the salt crust. 
ANÁLISIS ESTADÍSTICO

Se usó el promedio final a los 50 días de cada parámetro como medida de crecimiento y desarrollo de las plántulas. Las diferencias de las medias en conductividad eléctrica, concentración de sodio y cloruro, densidad aparente y porosidad de los suelos experimentales, crecimiento de las raíces (longitud, biomasa, cociente raíz / tallo, longitud radicular específica), crecimiento aéreo y mortalidad de plántulas fueron analizadas separadamente con un ANDEVA de una vía. El incremento de biomasa radicular en el tiempo fue analizado usando un ANDEVA de medidas repetidas con el tiempo como factor dentro del sujeto y los sustratos experimentales como factor entre sujetos. Todos los valores de $P$ fueron ajustados a Huynh-Feldt, un procedimiento que corrige las desviaciones de la presunción de esfericidad de la matriz de varianza-covarianza (Zar 1999). El contenido de cloruro y sodio en el tejido de las plántulas fue analizado usando Bootstrap (Efron 1979, Efron \& Tibshirani 1994), una técnica útil para determinar pruebas de significancias e intervalos de confianza a partir de una pequeña muestra de datos. La normalidad de los datos se evaluó con test de Shapiro-Wilks y la homocedasticidad con test de Barlett (Zar 1999). Para las comparaciones a posteriori se usó comparaciones múltiples de t-test y los valores de probabilidad fueron ajustados usando Bonferroni. Los análisis fueron efectuados con software R 3.0.2 (R Development Core Team 2009).

\section{RESULTADOS}

SALINIDAD DE SUELOS EXPERIMENTALES

La conductividad eléctrica (C.E.) tuvo una caída significativa cuando se incorporó arena al suelo natural del Salar de Bellavista (ANDEVA una vía: $\mathrm{F}_{2,25}=18,79 ; \mathrm{P}<$ 0,001). En el suelo 1:1 el valor de C.E. resultante fue de $132 \mathrm{dS} / \mathrm{m}$ y en el suelo 1:2, de $140 \mathrm{dS} / \mathrm{m}$ (Tabla 2). En estos dos suelos también se detectó una menor concentración de sales comparado al suelo salino natural. La disminución de la concentración de sodio y cloruro en el suelo natural (2333 $\mathrm{mmol} / \mathrm{L}$ y $4143 \mathrm{mmol} / \mathrm{L}$, respectivamente) fue significativa en los suelos 1:1 y 1:2. El valor promedio en el contenido de sodio y cloruro en estos dos suelos fue $1480 \mathrm{mmol} / \mathrm{L}$ y 2429 $\mathrm{mmol} / \mathrm{L}$, respectivamente. Esto representó en promedio una caída porcentual de $37 \%( \pm 0,1)$ en sodio y $41 \%( \pm 2,5)$ en cloruro. La arena también promovió un cambio significativo en la densidad aparente de los suelos experimentales 1:1 y 1:2 (ANDEVA una vía: $\mathrm{F}_{2,27}=143,46 ; \mathrm{P}<0,0001$ ), presentando éstos los valores más alto que lo detectado en el suelo salino natural (Tabla 2). Este cambio significativo en la densidad aparente indicó un incremento en el volumen de espacios porosos en los suelos 1:1 y 1:2.
CReCimiento de las Raíces EN LOS SUELOS EXPERIMENTALES Las plántulas que crecieron en los suelos experimentales 1:1 y 1:2 presentaron el mayor crecimiento radicular después de 50 días (Tabla 3 y Fig. 2 y 3). En estas plántulas se detectó un incremento significativo de la longitud radicular (ANDEVA una vía: $\left.\mathrm{F}_{2,27}=22,26 ; \mathrm{P}<0,0001\right)$, biomasa radicular $\left(\mathrm{F}_{2,27}=\right.$ $12,28 ; \mathrm{P}=0,0002) \mathrm{y}$ biomasa total $\left(\mathrm{F}_{2,27}=18,49 ; \mathrm{P}<0,0001\right)$ (Tabla 3). El aumento en la biomasa seca de raíz $(\Delta)$ mostró un cambio significativo sólo en las plántulas que crecieron en los suelos 1:1 y 1:2 (ANDEVA de medidas repetidas $\left(\mathrm{F}_{2}\right.$, $\left.{ }_{58}=12,02 ; \mathrm{P}<0,0001\right)$ a partir de los 30 días de iniciado el estudio (Fig. 2). Las plántulas que crecieron en los suelos $1: 1$ y $1: 2$ también presentaron un mayor cociente raíz / tallo $(\mathrm{R} / \mathrm{T})$ y longitud radicular específica (LRE) (ANDEVA una vía: $\mathrm{F}_{2,27}=11,80 ; \mathrm{P}=0,0002$ y $\mathrm{F}_{2,27}=56,94 ; \mathrm{P}<0,0001$, respectivamente). Aquí se detectaron diferencias de hasta tres veces en el valor promedio de $\mathrm{R} / \mathrm{T}(0,39 \pm 0,018)$ y cinco veces en el valor de LRE (25991 \pm 1385$)$ comparado a las plántulas que crecieron en suelo salino natural $(0,11 \pm$ 0,020 y $5141 \pm 1058$, respectivamente) (Fig. 3).

Mortalidad DE PlÁNTUlas Y CRECIMIENTO AÉREO EN LOS SUELOS EXPERIMENTALES

La mortalidad de plántulas fue significativamente menor en los suelos experimentales 1:1 y 1:2 comparado con aquellas plántulas que crecieron en suelo salino natural (ANDEVA de una vía: $\mathrm{F}_{1,7}=43,89 ; \mathrm{P}<0,0002$ ). Además, hubo mayor crecimiento aéreo con mayor desarrollo de hojas verdaderas en las plántulas que crecieron en los suelos que fueron mezclados con arena, en particular, el suelo 1:2 (ANDEVA de una vía: $\mathrm{F}_{1,88}=6,27 ; \mathrm{P}=0,0140$ ) (Tabla 3).

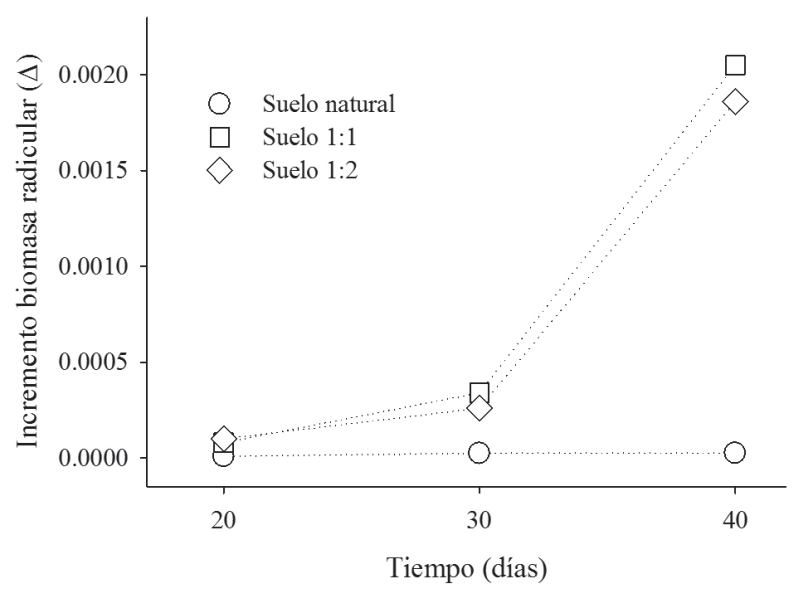

FIGURA 2. Incremento temporal en la producción de biomasa seca raíz en plántulas de Prosopis tamarugo determinadas a los 20, 30 y 40 días en el presente estudio. Los valores son deltas obtenidos de las medias de cada período evaluado ( $\mathrm{n}=10$ plántulas). / Temporal increasing in the production of dry root biomass in Prosopis tamarugo seedlings determined at 20,30 and 40 days in the present study $(\mathrm{n}=$ 10 seedlings). 
Tabla 3. Crecimiento y desarrollo de raíces, hojas y mortalidad final a los 50 días en plántulas de Prosopis tamarugo creciendo en tres suelos experimentales. Los valores son promedio $\pm(1 \mathrm{EE}, \mathrm{n}=10$ plántulas), excepto el número de hojas $(\mathrm{n}=30)$. Letras diferentes corresponden a diferencias significativas (Comparaciones múltiples t-test, $\mathrm{P}<0,001$ ). / Growth and development of roots and leaves and final mortality at day 50 in seedlings of Prosopis tamarugo growing in three experimental soils. Values are mean $\pm(1 \mathrm{EE}, \mathrm{n}=10 \mathrm{seedlings})$, except the number of leaves $(\mathrm{n}=30)$. Different letters correspond to significant differences (t-test multiple comparisons, $\mathrm{P}<0.001)$.

\begin{tabular}{|c|c|c|c|c|}
\hline SUELOS EXPERIMENTALES & LONGITUD RAÍZ $(\mathrm{cm})$ & BiomASA RAÍZ (g) & $\begin{array}{c}\text { PLÁNTULAS CON } 4^{\mathrm{TO}} \text { PAR DE } \\
\text { HOJAS }(\%)\end{array}$ & $\begin{array}{c}\text { MORTALIDAD PLÁNTULAS } \\
(\%)\end{array}$ \\
\hline Suelo natural & $3,5^{\mathrm{a}} \pm 7,86$ & $0,001^{\mathrm{a}} \pm 0,0001$ & $58,3^{\mathrm{a}} \pm 0,27$ & $15,3^{\mathrm{a}} \pm 0,88$ \\
\hline Suelo 1:1 & $9,1^{b} \pm 11,62$ & $0,003^{\mathrm{b}} \pm 0,0003$ & $69,6^{\mathrm{ab}} \pm 0,24$ & $6,6^{\mathrm{b}} \pm 0,33$ \\
\hline Suelo 1:2 & $9,9^{b} \pm 11,22$ & $0,004^{b} \pm 0,0004$ & $74,2^{b} \pm 0,22$ & $3,3^{\mathrm{b}} \pm 0,33$ \\
\hline
\end{tabular}
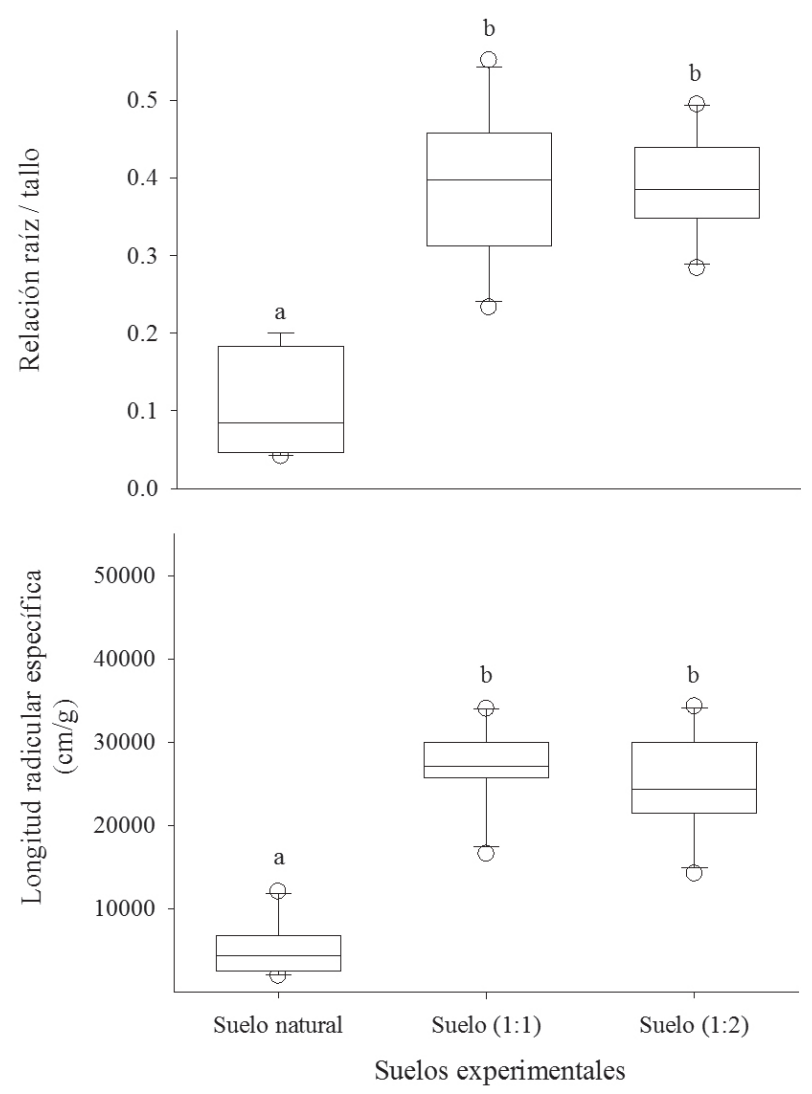

Figura 3. Distribución de datos para Proporción raíz / tallo y longitud radicular específica medida a los 50 días en plántulas de Prosopis tamarugo creciendo en tres suelos experimentales. Letras diferentes corresponden a diferencias significativas (Comparaciones múltiples t-test, $\mathrm{P}<0.001)$. / Data distribution for root / stem ratio and specific root length measured at 50 days in Prosopis tamarugo seedlings growing on three experimental soils. Different letters correspond to significant differences (t-test multiple comparisons, $\mathrm{P}<0.001$ ).

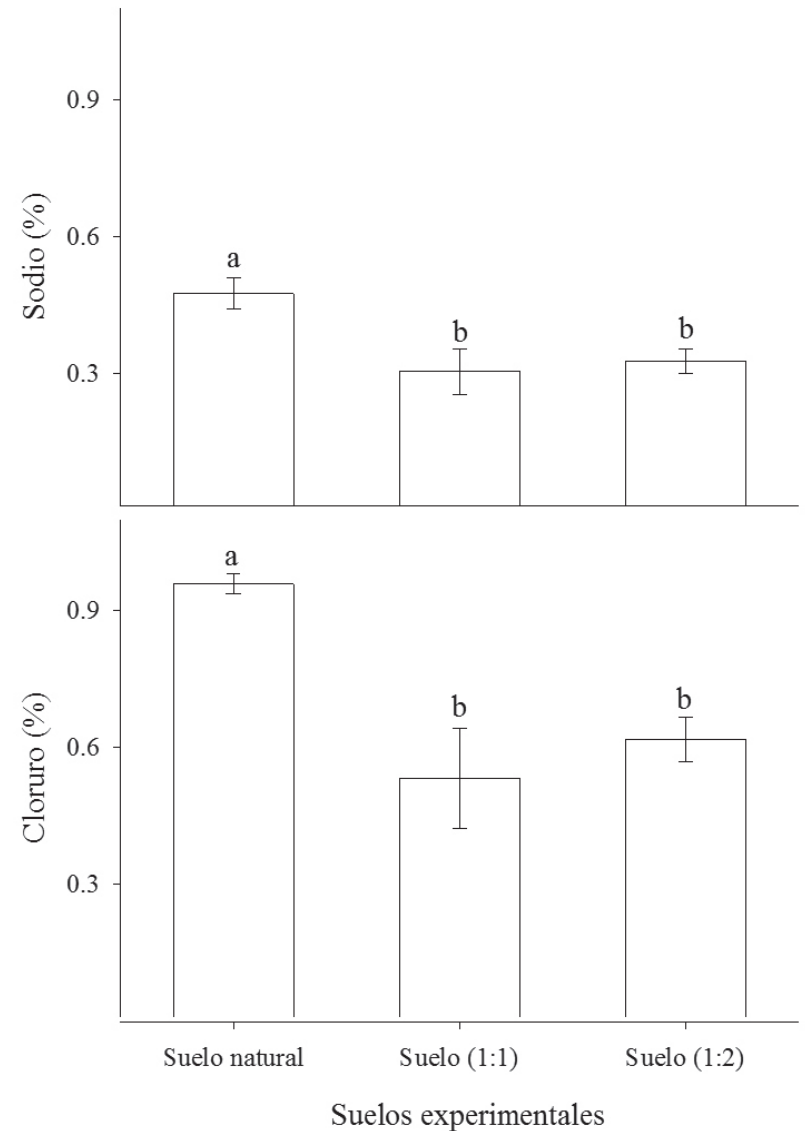

FIgURA 4. Porcentaje de sodio y cloruro foliar medido a los 50 días en plántulas de Prosopis tamarugo creciendo en tres suelos experimentales. Los valores son promedios $( \pm 1 \mathrm{EE}, \mathrm{n}=3)$. Letras iguales indican superposición de los intervalos de confianza con 95\%. / Percentage of sodium and chloride in leaves of seedlings of Prosopis tamarugo at 50 days of growing in three experimental soils. Values are mean $( \pm 1 \mathrm{SE}, \mathrm{n}=3)$. Equal letters indicate overlapping confidence intervals of $95 \%$. 
SALINIDAD EN LOS TEJidOS DE LAS PLÁNTULAS

El contenido de sodio y cloruro en los tejidos de las plántulas que crecieron en los suelos experimentales $1: 1$ y $1: 2$ fue estadísticamente diferente comparado con las plántulas que crecieron en suelo salino natural (Fig. 4). El bootstrap para el contenido de sodio y cloruro en los tejidos de las plántulas que crecieron en el suelo 1:1 fue significativamente menor que el detectado en las plántulas que crecieron en suelo salino natural, $\mathrm{B}_{\text {sodio }}=0,17, \mathrm{P}<0,05,95 \%$ intervalo de confianza $[-0,164,0,167]$ y $\mathrm{B}_{\text {cloruro }}=0,41, \mathrm{P}<0,05,95 \%$ IC $[-0,378,0,378]$. Para las plántulas que crecieron en el suelo $1: 2$, el procedimiento bootstrap nuevamente mostró diferencias significativas en el contenido de sodio y cloruro comparado con las plántulas que crecieron en suelo salino natural, $\mathrm{B}_{\text {sodio }}=0,14, \mathrm{P}<0,05,95 \%$ IC $[-0,136,0,134] \mathrm{y}$ $\mathrm{B}_{\text {cloruro }}=0,33, \mathrm{P}<0,05,95 \%$ IC $[-0,274,0,274]$. Cuando se comparó el contenido de sodio y cloruro de las plántulas que crecieron en el suelo 1:1 y 1:2 no se detectaron diferencias significativas, $\mathrm{B}_{\text {sodio }}=-0,02, \mathrm{P}>0,05,95 \%$ IC $[-0,087$, $0,090]$ y $\mathrm{B}_{\text {cloruro }}=-0,08, \mathrm{P}>0,05,95 \%$ IC $[-0,207,0,202]$ (Fig. 4).

\section{DISCUSIÓN}

La salinidad del suelo natural produjo un efecto negativo en el crecimiento y la supervivencia de plántulas de Prosopis tamarugo. Este suelo presentó un valor inicial de conductividad eléctrica de $176 \mathrm{dS} / \mathrm{m}$ y un alto nivel de sodio y cloruro los cuales, incorporados en los tejidos de las plántulas, produjeron un crecimiento irregular de sus raíces y crecimiento aéreo causando tempranamente su mortalidad en el estudio. Los estados aquí descritos reflejan una situación de colapso de las funciones fisiológicas en las plántulas por acción de estos iones salinos en algunos de los tres efectos que éstas suelen ejercerlo (e.g., efecto osmótico, toxicidad por excesiva absorción de sodio y cloro y desbalance nutricional (Munns 2002, Munns \& Tester 2008, Reginato et al. 2014). Sin embargo, el uso de los suelos experimentales $(1: 1$ y 1:2) produjo una caída de la conductividad eléctrica en un valor promedio general de 136 $\mathrm{dS} / \mathrm{m}$ con una disminución notable de las concentraciones de sodio y cloruro (37 y $41 \%$, respectivamente) en el momento que las semillas de tamarugo fueron sembradas. Aunque se produjo un valor de conductividad eléctrica resultante todavía alto, no impidió el progreso de las semillas germinadas el desarrollo de plántulas de $P$. tamarugo, denotando su capacidad para soportar este nivel salino más adecuado para su desempeño. Son pocas las plantas capaces de tolerar un umbral de salinidad de $136 \mathrm{dS} / \mathrm{m}$ (U.S. Salinity Laboratory Staff 1954), pero en situaciones experimentales controladas, $P$. tamarugo ha mostrado una respuesta similar (Felker et al. 1981, Arce et al. 1990, Serrato et al. 1991), algo que también es común dentro del género, puesto que otras especies exhiben una notable tolerancia a la salinidad en condiciones controladas como en $P$. cineraria (L.) Druce (Serrato et al. 1992), P. chilensis (Molina) Stuntz (Cazebonne et al. 1999) y P. strombulifera (Lam.) Benth. (Reinoso et al. 2004). Los resultados también concuerdan con el rápido efecto nocivo de las sales en el crecimiento y desarrollo de las plántulas de tamarugo (Serrato et al. 1992, Reinoso et al. 2004). Además del bajo número de pares de hojas formadas (cuarto par), acompañado por restricciones en la expansión de cotiledones y "acortamiento" de entrenudos (sólo observaciones), las alteraciones en la estructura de las raíces de las plántulas fueron sustanciales. Entre éstas, cambios en la longitud y biomasa radicular, un menor cociente raíz/ tallo y una baja longitud radicular específica. Estos cambios en el crecimiento de las raíces fueron determinantes en la mortalidad detectada en las plántulas de $P$. tamarugo.

Al tratarse de una especie freatófita, las plántulas de $P$. tamarugo estimulan tempranamente el crecimiento de raíces pivotantes en los estratos profundos del suelo, de este modo logran acceder al agua presente en las napas subterráneas (Burgess et al. 2000, 2001). Esta asignación importante de recursos al crecimiento de sus raíces facilita la obtención rápida de recursos disponibles aumentando sus probabilidades de establecimiento como es descrito para algunas plantas perennes del semiárido de Chile (León et al. 2011). Entonces, las oportunidades que tiene $P$. tamarugo para facilitar el crecimiento de sus raíces sólo pueden suscitarse cuando el nivel de salinidad del suelo in situ disminuye a una fracción tolerable para las plántulas, cuando se producen escorrentías causadas por eventos episódicos estivales en la cordillera. En el estudio se constató que concentraciones extremadamente altas del suelo natural son perjudiciales para el establecimiento efectivo de plántulas de $P$. tamarugo y, como en los suelos 1:1 y 1:2 donde el crecimiento radicular de las plántulas fue notable, disminuciones leves pero significativas del contenido de iones en el suelo ayudan al establecimiento exitoso de plántulas de esta especie.

En el manejo de la salinidad del suelo es relevante impedir la interacción de los iones salinos con las raíces de las plantas de modo de no favorecer su absorción. A través de cambios en algunas propiedades del suelo por medio de métodos que han sido creados para estos propósitos, la presencia de sales puede ser contrarrestada (Marschner 1995) (aplicación de enmiendas Gurbachan et al. 1989, Diacono \& Montemurro 2015, aplicación de nutrientes Hussain et al. 2015). Aquí avanzamos en la creación de un suelo experimental al utilizar arena en mezcla con el suelo salino natural, lo que permitió aumentar su densidad aparente. El aumento del volumen de espacios porosos junto a una disminución paulatina de la proporción de poros finos alteró la concentración iones salinos disminuyendo su presencia en la zona de las raíces. Los suelos experimentales estudiados aquí representan un método simple y económico 
para desafectar la presencia de sales del suelo, nosotros apoyamos esta idea debido a los resultados favorables en la supervivencia de plántulas. La factibilidad de uso del sustrato en estudio para futuras plantaciones experimentales de plantas juveniles de $P$. tamarugo podría ser promisorio en su hábitat natural debido a que la disminución de la conductividad eléctrica y concentración de sodio y cloruro es una condición permanente cuando se mezcla arena con el suelo salino (Chile Forestal, en prensa).

P. tamarugo es la principal especie de Pampa del Tamarugal, tanto en plantaciones como en poblaciones naturales. Constituye la base en la provisión de servicios ecosistémicos en la que se sustentan los niveles tróficos superiores y la actividad ganadera realizada por las comunidades locales. $\mathrm{Su}$ preservación requiere un esfuerzo económico y humano urgente debido a la falta de regeneración en su hábitat natural, pero incrementar su densidad poblacional en cualquiera de los sectores que conforman la reserva requiere superar la carga salina del suelo por medio de métodos efectivos y económicamente viables. A la variedad de métodos existentes para manejar la salinidad, nosotros agregamos la creación de los sustratos en estudio para este objetivo.

\section{CONCLUSIONES}

A pesar de la habilidad para tolerar la salinidad, la sensibilidad de las raíces en los estados iniciales de Prosopis tamarugo a la presencia de iones salinos, tiene implicancias directas con su regeneración natural y en consecuencia, reafirma la preocupación de su estatus de conservación en la condición actual de la especie en su hábitat. Los resultados usando suelos experimentales, son aquí una vía plausible simple para manejar sustentablemente la salinidad del suelo en la Reserva Nacional Pampa del Tamarugal.

\section{AGRADECIMIENTOS}

A Sociedad Química y Minera de Chile S.A (SQM). La investigación fue parte del Plan de Manejo Ambiental de Tamarugo, Proyecto Pampa Hermosa. Agradecemos la asistencia técnica de María José Espejo, Ismael Jiménez y Eric Ibacache en el estudio.

\section{REFERENCIAS}

Altamirano, H. 2006. Prosopis tamarugo Phil. Tamarugo. En: Donoso Zegers, C. (ed.), Las especies arbóreas de los bosques templados de Chile y Argentina, pp. 534-540. Marisa Cuneo Ediciones, Valdivia, Chile.

Arce, P., Medina, C., Balboa, O. 1990. Tolerancia a la salinidad en tres especies de Prosopis ( $P$. alba, P. chilensis y $P$. tamarugo). Ciencia e Investigación Agraria 17(1-2): 7175.

ASTM D2216-10. 2010. Standard test methods for laboratory determination of water (moisture) content of soil and rock by mass, ASTM International, West Conshohocken, Pennsylvania. $7 \mathrm{pp}$.

Bradbury, M., Ahmad, R. 1990. The effect of silicon on the growth of Prosopis juliflora growing in saline soil. Plant and Soil 125(1): 71-74.

Burgess, S.S.O., Adams, M.A., Turner, N.C., White, D.A., Ong, C.K. 2001. Tree roots-conduits for deep recharge of soil water. Oecologia 126(2):158-165.

Burgess, S.S.O., Pate, J.S., Adams, M.A., Dawson, T.E. 2000. Seasonal water acquisition and redistribution in the Australian woody phreatophyte, Banksia prionotes. Annals of Botany 85(2): 215-224.

Burkart, A. 1976. A monograph of the genus Prosopis (Leguminosae subfam. Mimosoideae). Journal of the Arnold Arboretum 57(3): 219-249.

CAREvic, F.S. 2014. The role of ecophysiological studies in the genus Prosopis: implications for the conservation of drought-prone species. IDESIA 32(4): 77-81.

Carevic, F.S., Carevic, A., Delatorre, J. 2012. Historia natural del género Prosopis en la Región de Tarapacá. IDESIA 30(3): 113-117.

Catalán, L., Balzarini, M., Taleisnik, E., Sereno, R. 1994. Effects of salinity on germination and seedling growth of Prosopis flexuosa (DC.). Forest Ecology and Management 63(2-3): 347-357.

Cazebonne, C., Vega, A.I., Varela, D.A., Cardemil, L.A. 1999. Salinity effects on germination and growth of Prosopis chilensis. Revista Chilena Historia Natural 72(1): 83-91.

Diacono, M., Montemurro, F. 2015. Effectiveness of organic wastes as fertilizers and amendments in salt-affected soils. Agriculture 5: 221-222.

Dixon, R.K., GARG, V.K., RAO, M.V. 1993. Inoculation of Leucaena and Prosopis seedlings with Glomus and Rhizobium species in saline soil: Rhizosphere relations and seedling growth. Arid Soil Research and Rehabilitation 7(2): 133-144.

EFron, B. 1979. Bootstrap methods: another look at the jackknife. Annals of Statistics 7(1): 1-26.

EFron, B., Tibshirani, R.J. 1994. An introduction to the bootstrap. CRC Press, New York. 456 pp.

Felker, P., Clark, P.R., Laag, A.E., Pratt, P.F. 1981. Salinity tolerance of the tree legumes: mesquite (Prosopis glandulosa var. torryana, P. velutina and P. articulata) algarrobo ( $P$. chilensis), kiawe (P. pallida) and tamarugo ( $P$. tamarugo) grown in sand culture on nitrogen-free media. Plant and Soil 61(3): 311-317.

Grattan, S.R., Grieve, C.M. 1992. Mineral element acquisition and growth response of plants grown in saline environments. Agriculture, Ecosystems \& Environment 38(4): 275-300.

Gurbachan, S., Abrol, I.P., Cheema, S.S. 1989. Effects of gympsum application on mesquite (Prosopis juliflora) and soil properties in an abandoned sodic soil. Forest Ecology and Management 29(1-2): 1-14.

Hosseini Nasr, S.M., Parsakhoo, A., Naghavi, H., Savadkoohi, S.K. 2012. Effect of salt stress on germination and seedling growth of Prosopis juliflora (Sw.). New Forests 43(1):4555. 
Houston, J. 2006. Evaporation in the Atacama Desert: an empirical study of spatio-temporal variations and their causes. Journal of Hydrology 330(3-4): 402-412.

Hurlbert, S.H. 1984. Pseudoreplication and the design of the ecological field experiments. Ecological Monographs 54(2): 187-211.

Hussain. Z., Khattak, R.A., Fareed, I., Irshad, M., Mahmood, Q. 2015. Interaction of phosphorus and potassium on maize in saline sodic soil. Journal of Agricultural Science 7(3): 66-78.

Huy, Y., Schmidhalter, U. 2005. Drought and salinity: a comparison of their effects on mineral nutrition of plants. Journal of Plant Nutrition and Soil Science 168(4): 541549.

Imada, S., Matsuo, N., Acharva, K., Yamanaka, N. 2015. Effects of salinity on fine root distribution and whole plant biomass of Tamarix ramosissima cuttings. Journal of Arid Environments 114: 84-90.

KRAMER, P.J., BOYER, J.S. 1995. Water relations of plants and soils. Academic Press, San Diego. 495 pp.

León, M.F., Squeo, F.A., Gutiérrez, J.R., Holmgren, M. 2011. Rapid root extension during water pulses enhances establishment of shrub seedlings in the Atacama Desert. Journal of Vegetation Science 22(1): 120-129.

Marschner, H. 1995. Mineral nutrition of higher plants. Academic Press, San Diego. 889 pp.

Mer, R.K., Prajith, P.K., Pandya, D.H., Pandey, A.N. 2000. Effect of salts on germination of seeds and growth of young plants of Hordeum vulgare, Triticum aestivum, Cicer arietinum and Brassica juncea. Journal of Agronomy and Crop Science 185(4): 209-217.

Ministerio del Medio Ambiente. 2013. Información procesos de clasificación de especies. URL: http://www.mma. gob.cl/clasificacionespecies/fichas9proceso/FICHAS INICIO_9o_PROCESO_PDF/Prosopis_tamarugo.pdf. Visitado: Septiembre 23, 2015.

Mooney, H.A., Gulmon, S.L., Rundel, P.W., Ehleringer, J.H. 1980. Further observations on the water relations of Prosopis tamarugo of the northern Atacama Desert. Oecologia 44(2): 177-180.

MunNs, R. 2002. Comparative physiology of salt and water stress. Plant, Cell and Environment 25(2): 239-250.
Munns, R., Tester, M. 2008. Mechanisms of salinity tolerant. Annual Review of Plant Biology 59: 651-681.

R Development Core team. 2009. R: A language and environment for statistical computing. R Foundation for statistical computing, Vienna, Austria. ISBN 3-900051-07-0, URL http://www.R-project.org.

Reinoso, H., Sosa, L., Ramírez, L., Luna, V. 2004. Saltinduced changes in the vegetative anatomy of Prosopis strombulifera (Leguminosae). Canadian Journal of Botany 82(5): 618-628.

Reginato, M.A., Castagna, A., Furlán, A., Castro, S., Ranieri, A., LunA, V. 2014. Physiological responses of a halophytic shrub to salt stress by $\mathrm{Na}_{2} \mathrm{SO}_{4}$ and $\mathrm{NaCl}$ : oxidative damage and the role of polyphenols in antioxidant protection. AoB PLANTS 6: plu 042.

Sadzawka, R.A., Carrasco, M.A., Demanet, R., Flores, H., Grez, R., Mora, M.L., Neaman, A. 2007. Métodos de análisis de tejidos vegetales. Segunda Edición. Instituto de Investigaciones Agropecuarias. Series de Actas INIA N ${ }^{\circ}$ 40, Santiago, Chile. 140 pp.

Serrato, V.G., Ferro, M., Ferraro, D., Riveros, F. 1991. Anatomical changes in Prosopis tamarugo Phil. seedlings growing at different levels of $\mathrm{NaCl}$ salinity. Annals of Botany 68(1): 47-53.

Serrato, V.G., Melone, L., Orsi, O., Riveros, F. 1992. Anatomical changes in Prosopis cineraria (L.) Druce seedlings growing at different levels of $\mathrm{NaCl}$ salinity. Annals of Botany 70(5): 399-404.

SinHA, B.K., SingH, N.T. 1974. Effect of transpiration rate on salt accumulation around corn roots in a saline soil. Agronomy Journal 66(4): 557-560.

Sosa, L., Llanes, A., Reinoso, H., Reginato, M., Luna, V. 2005. Osmotic and specific ion effects on the germination of Prosopis strombulifera. Annals of Botany 96(2): 261-267

Yang, Y., Liu, X., Jiang, Y., Xiang, Z., Xu, Q., Zhao, N., Shu, B. 2015. Root growth, free amino acids, and carbohydrates of tall fescue in response to soil salinity. HortScience 50(4): 609-614

ZAR, J.H. 1999. Biostatistical analysis. Prentice Hall, Upper Saddle River, NJ. 662 pp.

Recibido: 09.12.2015

Aceptado: 19.12.2016 